\title{
Programming of the Development of Tumor-Promoting Neutrophils by Mesenchymal Stromal Cells
}

\author{
Xiaoyu Hu ${ }^{a}$ Yushan Zhou ${ }^{b}$ Kui Dong ${ }^{c}$ Zhina Sun ${ }^{a}$ Dan Zhao ${ }^{a}$ Weiqiang Wang ${ }^{c}$ \\ Gang Yu ${ }^{b}$ Wentian Liu ${ }^{c}$ Guogang Xub,d Zhongchao Han ${ }^{\mathrm{a}}$ Xiaoming Feng $^{\mathrm{a}}$
}

aState Key Laboratory of Experimental Hematology, Institute of Hematology and Hospital of Blood Disease, Chinese Academy of Medical Sciences, Tianjin, bepartment of Respiratory Medicine, The Second affiliated hospital of Nanchang University, Nanchang, Jiangxi, 'Department of Gastroenterology and Hepatology, General Hospital, Tianjin Medical University, Tianjin, dNanlou Department of Pulmonary Medicine, Beijing 301 Hospital, Beijing, P.R. China

\section{Key Words}

Mesenchymal stromal cells • Neutrophils $・$ Immunosuppressive $・$ Tumor

\begin{abstract}
Background/Aims: Neutrophils obtain immunosuppressive function during tumor development, yet the mechanisms are largely unknown. This study explored whether and how mesenchymal stromal cells (MSCs), the key component of tumor microenvironment, regulate the suppressive function of neutrophils. Methods: Immunosuppressive function of neutrophils was evaluated by T cell proliferation assay and 4T1 breast tumor model; molecular mechanisms were explored by transcriptional profiling, Real-time RT-PCR, arginase activity assay, and iNOS inhibition experiments. Results: After being cocultured with MSCs primed by TNF- $\alpha$ (TNFMSCs), CD11 b+ Ly6G ${ }^{+}$neutrophils isolated from bone marrow of normal mice or spleen of tumorbearing mice obtained immunosuppressive function to inhibit T cell proliferation in vitro, and to enhance 4T1 tumor progression in vivo. Moreover, arginase activity and expression of iNOS, saa3, some cytokines and chemokines and their receptors, were upregulated in neutrophils after co-culture with TNF-MSCs. Inhibition of iNOS activity attenuated the suppressive effect of TNF-MSC pre-cocultured neutrophils on T cell proliferation. Conclusion: MSCs program neutrophils into an immunosuppressive and tumor-promoting phenotype.
\end{abstract}




\section{Introduction}

Mesenchymal stromal cells (MSCs) have potent regenerative capacity and serve as a major niche component for hematopoietic stem/progenitor cells in the bone marrow [1, $2]$. MSCs also possess potent immunomodulatory properties $[3,4]$ and they have been shown to interact with a variety of immune cells including T cells, B cells, dendritic cells, and monocytes/macrophages. MSCs are one of the key elements of tumor microenvironment and are also believed to be the precursors of tumor-associated fibroblasts [5-8]. Recent studies demonstrated that MSCs constitutively secreted a large number of cytokines, and contributed to greater recruitment of $\mathrm{CD} 11 \mathrm{~b}^{+} \mathrm{Ly} 6 \mathrm{C}^{+} \mathrm{Ly} 6 \mathrm{G}^{-}$monocytes, $\mathrm{F} 4 / 80+$ macrophages, and $\mathrm{CD} 11 \mathrm{~b}^{+} \mathrm{Ly} 6 \mathrm{C}^{\mathrm{dim}} \mathrm{Ly} 6 \mathrm{G}^{+}$neutrophils to the tumor to contribute to tumor growth [9]. In addition, it is also demonstrated that MSCs are capable of skewing monocytes/macrophages into an immunosuppressive phenotype, which might promote tumor progression [10].

Neutrophils as important effector cells in the innate immune response against invading micro-organisms [11] are always abundant in tumor microenvironment, yet their impact on tumor development still remains elusive. It has been recently recognized that neutrophils could display multiple function phenotype. In fact, only after priming (typically by a cytokine, chemokine or bioactive lipid) can neutrophils optimally exert pro-inflammatory functions such as the generation of a respiratory burst induced by fMLF or chemotaxis [12, 13]. In addition, neutrophils can be switched from non-suppressive to immunosuppressive phenotypes. Phenotype switching of neutrophils has recently been reported by the addition of granulocyte macrophage colony-stimulating factor (GM-CSF) to mature or immature murine bone marrow-derived neutrophils [14]. Recent studies also showed that tumorassociated neutrophils is driven by TGF- $\beta$ to switch to the tumor-promoting phenotype [15]. Neverthelss, it is still poorly understood how neutrophils switch into the suppressive phenotypes, especially during cancer development [16].

MSCs have been reported to promote the survival of neutrophils [17], yet the direct effect of MSCs on the function of neutrophils has not been addressed. Due to the key role of MSCs in promoting tumor progression in many conditions, we hypothesize that neutrophils are a major type of cells through which MSCs might exert their tumor-promoting effect. In this study, we investigated the effect of MSCs on the immunophenotype and functional characteristics of $\mathrm{CD} 11 \mathrm{~b}^{+} \mathrm{Ly}_{6 \mathrm{G}} \mathrm{G}^{+}$neutrophils, regarding its impact on tumor progression.

\section{Materials and Methods}

\section{Animals}

All animals were maintained in specific pathogen-free barrier facilities and were used in accordance with protocols approved by Animal Care and User Committee at the Institute of Hematology, Chinese Academy of Medical Sciences. 6-8 weeks old Balb/C mice were purchased from Chinese Academy of Medical Sciences, and used in all experiments.

Reagents

Recombinant mouse TNF- $\alpha$ was purchased from R\&D Systems. Antibodies including CD11b-PE, Ly6GAPC, CD25-APC and CD69-PE-Cy7 were from eBioscience or Biolegend.

\section{Cell culture}

Isolation and culture of bone marrow (BM) MSCs were carried out as previously described [18, 19] with some modifications. Briefly, animals were sacrificed by cervical dislocation and the femurs were flushed with complete medium (CM) containing DMEM/Ham's F-12 medium (Gibco), 10\% fetal bovine serum (Hyclone), $50 \mathrm{U} / \mathrm{ml}$ penicillin/streptomycin (Gibco). After filtering through a 60 micron nylon mesh to remove debris, cells were washed twice in Hanks' balanced salt solution and plated in a $25-\mathrm{cm}^{2} \mathrm{flask}$ at a density of $2-4 \times 10^{6}$ cells $/ \mathrm{cm}^{2}$ in $\mathrm{CM}$ and were incubated in a humidified $5 \% \mathrm{CO}_{2}$ incubator at $37^{\circ} \mathrm{C}$ for $72 \mathrm{~h}$. Non-adherent cells were removed by replacing the media after $72 \mathrm{~h}$. The media were replaced every 3 days. 


\section{Cellular Physiology Cell Physiol Biochem 2014;33:1802-1814 \begin{tabular}{l|l} 
and Biochemistry Publisned onlIne: Ivay 27, 2014 & $\begin{array}{l}\text { C) 2014 S. Karger AG, Basel } \\
\text { www.karger.com/cpb }\end{array}$ \\
\hline
\end{tabular}}

Hu et al.: MSCs Induces Tumor-Promoting Neutrophils

Confluent primary cultures were dissociated in 0.25\% Trypsin-EDTA solution (Gibco) and the cells were replated in $75-\mathrm{cm}^{2}$ culture flasks at a dilution of 1:3. MSCs in less than passage 3 were used in experiments.

The murine breast cancer cell line 4T1 was purchased from National Platform of Experimental Cell Resources for Sci-Tech and cultured in 1640 medium supplement with 10\% fetal bovine serum (Gibco).

Flow cytometry analysis

Cells were counted by Trypan Blue staining. Nonspecific antibody binding was blocked with antiCD16/CD32 antibodies (2.4G2) before staining. Cells were stained in staining buffer (PBS with 1\% BSA) with antibodies for $40 \mathrm{~min}$ at $4^{\circ} \mathrm{C}$, washed with staining buffer, and analyzed by LSRII (BD PharMingen). Data were analyzed by FlowJo software (Treestar).

\section{Isolation of neutrophils from bone marrow or spleen}

Bone marrow of normal Balb/C mice, or spleen of Balb/C mice bearing 4T1 tumors for 4 weeks, were harvested, filtered through a 60 micron nylon mesh, and red blood cells were lysed. The cells were stained in staining buffer with antibodies for $40 \mathrm{~min}$ at $4^{\circ} \mathrm{C}$, washed, and CD $11 \mathrm{~b}^{+} \mathrm{Ly} 6 \mathrm{G}^{+}$neutrophils were then sorted using a BD FACS Aria III flow sorter. The sorted populations were $>95 \%$ pure.

In vitro T cells proliferation assay

BM-MSCs were seeded at $2 \times 10^{5}$ cells/well in 24 well flat-bottom plates (BD Falcon) and cultured for $24 \mathrm{~h}$ at $37^{\circ} \mathrm{C}$, and stimulated with or without TNF- $\alpha$ for $24 \mathrm{~h}$. BM-MSCs were gently washed twice with fresh medium to remove any residual cytokine. Then sorted $\mathrm{CD} 11 \mathrm{~b}^{+} \mathrm{Ly}_{6} \mathrm{G}^{+}$neutrophils and MSC were co-cultured, at Neutrophil:MSC ratio of 10:1, for 48 to $72 \mathrm{~h}$ in complete medium.

Lymph node cells were obtained by filtering the homogenized lymph node through a 60 micron nylon mesh. For carboxyfluorescein succinimidyl ester (CFSE) labeling, lymph node cells were incubated at $10^{7} /$ $\mathrm{ml}$ with $2 \mu \mathrm{M}$ CFSE (Invitrogen) for 8 minutes at room temperature, then washed twice in medium with 10\% fetal bovine serum.

Lymph node cells labeled with CFSE were stimulated by anti-CD3 (145.2-C11) for $72 \mathrm{~h}$, without or with addition of fresh neutrophils, or MSC pre-cocultured neutrophils which were sorted to eliminate contamination of MSCs.

Microarray analysis

CD11b+Ly6G+ neutrophils from bone marrow of normal Balb/C mice, or spleen of tumor bearing mice were sorted as described above, and cocultured with TNF- $\alpha$ primed MSCs for the indicated time. At the end of coculturing, total RNA was extracted from the re-sorted CD11b+Ly6G+ cells with Trizol reagent (Invitrogen, Carlsbad, CA, USA). cDNA samples were hybridized to HumanGene 1.0 ST GeneChip arrays (Affymetrix, America) according to the User Manuals.Affymetrix Expression Console Software (version 1.2.1) was used for microarray analysis. Raw data (CEL files) were normalized at transcript level using robust multiaverage method (RMA workflow). Median summarization of transcript expressions was calculated. Gene level data was then filtered to include only those probe sets that are in the "core" metaprobe list, which represent RefSeq genes.

\section{Real-time RT-PCR}

Total cellular RNA of different samples was extracted the same as in microarray analysis, and Realtime RT-PCR was performed as described [20]. The primer pairs were as follows:

Saa3, forward 5'-TCTTTGCATCTTGATCCTGGG-3' and reverse 5'-CGAGCATGGAAGTATTTGTCTG-3'; Nos2, forward 5'-GCAAACATCACATTCAGATCCC-3' and reverse 5'-TCAGCCTCATGGTAAACACG-3'; IL1a, forward 5'-TGCAGTCCATAACCCATGATC-3' and reverse 5'-ACAAACTTCTGCCTGACGAG-3'; IL-10ra, forward 5'-CCTATCCCAAACCAGTCTGAG-3' and reverse 5'-GTGATACAGATCCAGGGTGAAC-3'; IL-4a, forward 5'-CTGTCTGATTTTGCTGTTGGTG-3' and reverse 5'-GATGTAGTCAGAGAAGCAGGTG-3'; IL10, forward 5'-AGCCGGGAAGACAATAACTG-3' and reverse 5'-GGAGTCGGTTAGCAGTATGTTG-3'; IL6, forward 5'-CAAAGCCAGAGTCCTTCAGAG-3' and reverse 5'-GTCCTTAGCCACTCCTTCTG-3'; Cxcl3, forward 5'-CCCCAGGCTTCAGATAATCAT-3' and reverse 5'-AAAGACACATCCAGACACCG-3'; Cxcr1, forward 5'-GTTCCAATAACCAACAGGCAG-3' and reverse 5'-TTCAGCACGTAGACATCCATG-3'. 
Arginase acitivity assay

Arginase activity was determined by a quantitative colorimetric assay at $430 \mathrm{~nm}$, employing a QuantiChrom arginase assay kit (Bioassay Systems) according to the manufacture's instructions. Briefly $1 \times 10^{5}$ cells per sample were harvested and washed with PBS, centrifuged, and cell pellet was lysed for 10 min in $100 \mu \mathrm{l}$ of $10 \mathrm{mM}$ Tris- $\mathrm{HCl}(\mathrm{pH}$ 7.4) containing $1 \mu \mathrm{M}$ pepstatin A, $1 \mu \mathrm{M}$ leupeptin, and $0.4 \%$ Triton $\mathrm{X}-100$. The lysate was centrifuged at $14,000 \mathrm{~g}$ at $4^{\circ} \mathrm{C}$ for $10 \mathrm{~min}$. Supernatant was mixed with $10 \mu \mathrm{l}$ of the substrate buffer and incubated at RT for $2 \mathrm{~h}$. Samples were then added to a 96-well flat-bottomed plate (Corning Inc) with the appropriate blank controls. Urea reagent (supplied with kit) was then added to the individual wells to stop the arginase reaction. After incubating at RT for another $15 \mathrm{~min}$, the absorbance was measured at $430 \mathrm{~nm}$ and enzyme activity (in IU) was calculated according to the kit instructions.

\section{Inhibition of iNOS activity}

S-methyl-isothiourea (SMT) (Sigma) was used as an selective iNOS inhibitor to explore the effect of iNOS activity on suppressive function of neutrophils. Neutrophils pre-cocultured with TNF-MSCs were cocultured with lymph node cells simulated by anti-CD3, supplemented with or without $1 \mathrm{mM}$ SMT, for 3 days. Then T cells proliferation was analyzed as described before.

Tumor model

$4 \mathrm{~T} 1$ cells $\left(2 \times 10^{4}\right)$ were injected into Balb/c mice in the intramammary gland fat pad. Neutrophils were coinjected as indicated. Tumor sizes were measured with a caliper at various time points. Each experimental group included at least five mice. All experiments were replicated at least three times.

Statistical analysis

Unpaired Student t-tests were used to statistically evaluate the difference of sample means among two groups. For comparing more than two groups, we used one sided ANOVA with appropriate post hoc testing. Differences were considered significant when $P<0.05$. Data are presented as mean \pm SEM.

\section{Results}

MSCs promoted survival of normal and tumor associated neutrophils in vitro

We first evaluated the effect of MSCs on survival of normal or tumor associated neutrophils. When cultured in medium alone, $\mathrm{CD} 11 \mathrm{~b}^{+} \mathrm{Ly}_{6 \mathrm{G}} \mathrm{G}^{+}$neutrophils sorted from bone marrow (BM) rapidly died, leaving nearly half of the cells viable after $72 \mathrm{~h}$. By contrast, MSC treated BM neutrophils showed dramatically increased survival, with $80 \%$ viability after 72 $\mathrm{h}$ of culture (Fig. 1A).

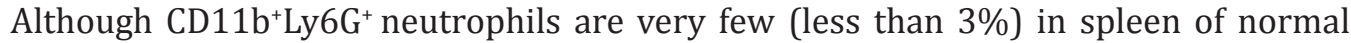
mice, their number dramatically increased (accounting for 20-30\% in total spleen cells) in the mice bearing 4T1 tumors. Similarly, MSC treatment promoted survival of CD11 b $\mathrm{b}^{+} \mathrm{Ly} 6 \mathrm{G}^{+}$ cells sorted from the spleen of mice bearing 4T1 tumors (Fig. 1B). These results suggest that MSC not only greatly promote survival of normal neutrophils as previously reported [17], but also potentiate survival of tumor associated neutrophils.

MSCs transform neutrophils into a T-cell-suppressive phenotype in vitro

First, the effect of MSCs on the immunosuppressive function of neutrophlis from BM of normal mice was examined. Fresh neutrophils (f-Neu), neutrophils cocultured with MSCs (MSCs-Neu), or neutrophils cocultured with TNF- $\alpha$ primed MSCs which mimic tumor-educated MSCs (TNF-MSCs-Neu), were added into lymphocytes stimulated by antiCD3 antibody to determine whether MSC treated neutrophils could inhibit T lymphocyte proliferation. Cell size by FSC, T cell proliferation by CFSE, and expression of $\mathrm{T}$ cell marker CD25 and CD69 (which were highly expressed in activated T cells) were examined by flow cytometry analysis.

Only weak changes of T lymphocyte activation were observed after being cocultured with f-Neu: CD4 ${ }^{+} \mathrm{T}$ cells showed slightly suppressed proliferation, FSC and CD25 expression, 
Fig. 1. MSCs promote survival of neutrophils in vitro. CD 11 b ${ }^{+}$Ly 6C ${ }^{\text {dim }}{\text { Ly } 6 G^{+}}^{+}$ neutrophils from BM or spleen of tumor-bearing mice were cocultured with BM MSCs for 72 hours and analyzed for (A) expression of Ly6C and Ly6G. (B) Annexin $\mathrm{V}$ and PI staining. Data are representative of two independent experiments.

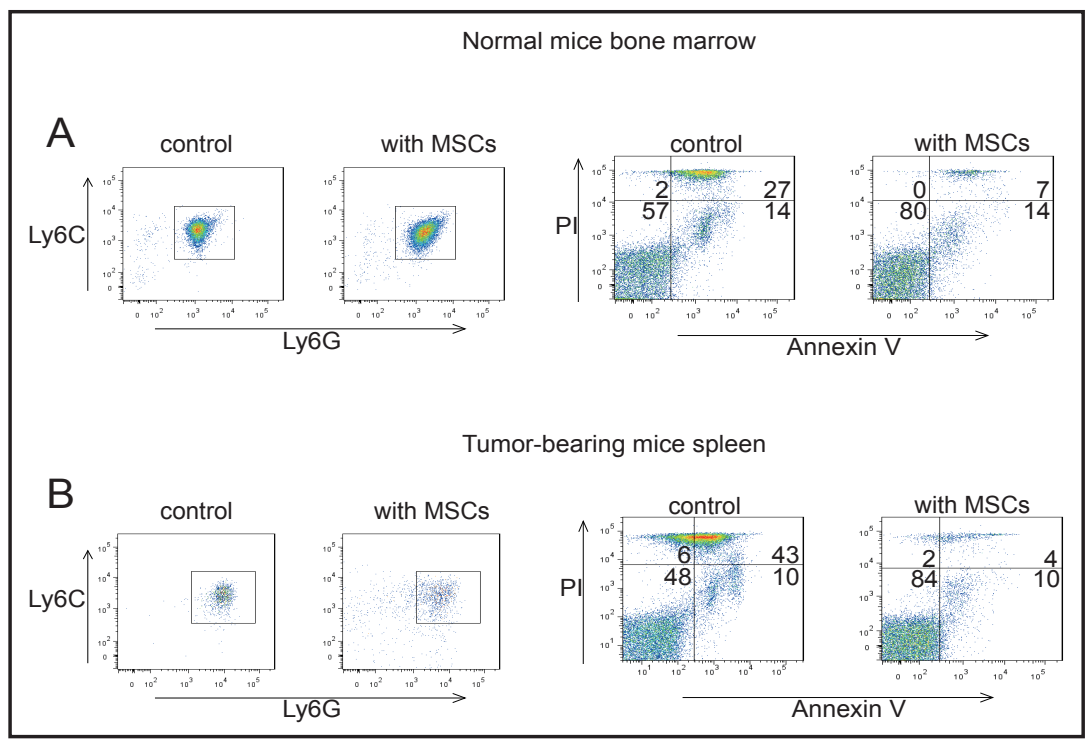

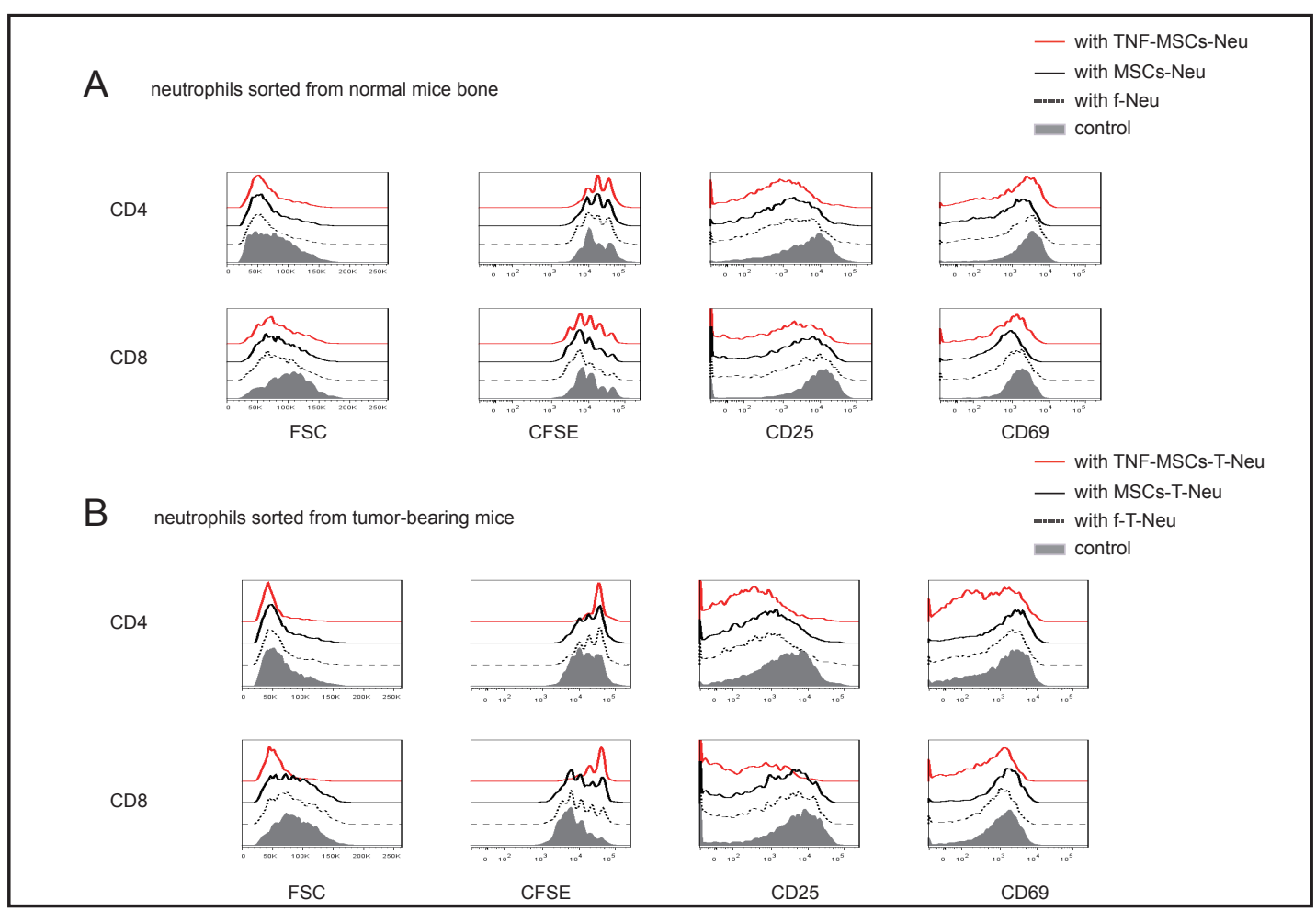

Fig. 2. MSCs transform neutrophils into an immunosuppressive phenotype in vitro. Cell size, CFSE proliferation profile, and expression of activation marker CD44, CD62L, and CD69 were analyzed by flow cytometry for (A) Lymph node cells that were stimulated with anti-CD3 antibody for 3 days, with or without the addition of BM neutrophils (f-Neu), MSC treated BM neutrophils (MSCs-Neu), or TNF-MSC treated neutrophils (TNF-MSCs-Neu), and (B) Lymph node cells that were stimulated with anti-CD3 antibody for 3 days, with or without the addition of tumor-spleen neutrophils (f-T-Neu), MSC-treated tumor-spleen neutrophils (MSCsT-Neu), or TNF-MSC treated tumor-spleen neutrophils (TNF-MSCs-T-Neu). Data are representative of three independent experiments.

but no changes in CD69 expression; CD8 ${ }^{+} \mathrm{T}$ cells showed slightly suppressed FSC and CD25 expression, but no changes in proliferation and CD69 expression. Except for suppression of CD69 expression on $\mathrm{CD}^{+}$and $\mathrm{CD}^{+} \mathrm{T}$ cells, no differences were found between $\mathrm{f}-\mathrm{Neu}$ and 
Fig. 3. MSC treated neutrophils promoted tumor progression in breast carcinoma 4T1 model. The tumor volume of 4T1 tumor-bearing mice treated by f$\mathrm{Neu}, \mathrm{MSCs}-\mathrm{Neu}$, or TNF-MSCs-Neu at day 15 (A), and at the indicated time points (B). Bars represent average \pm SEM. ${ }^{*} p<0.05$ vs $4 \mathrm{~T} 1$ control, and 5 mice per group were used. Data are representative of three independent
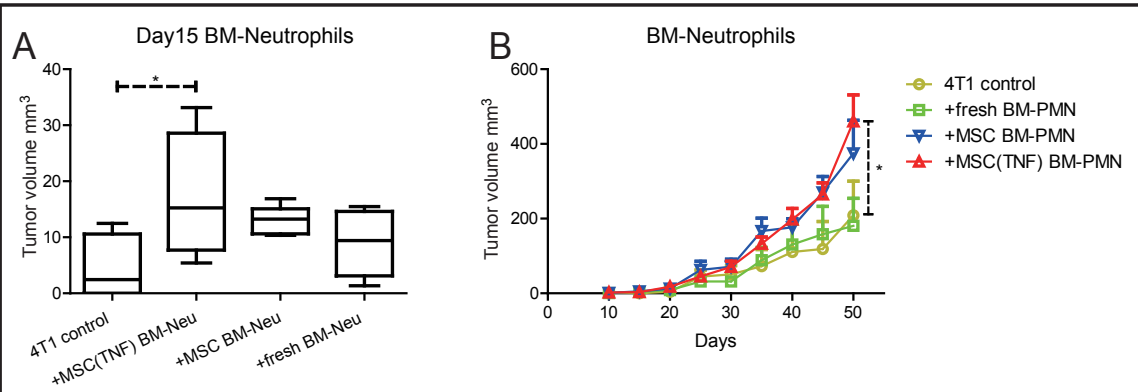

C Day15 Tumor-spleen-Neutrophils
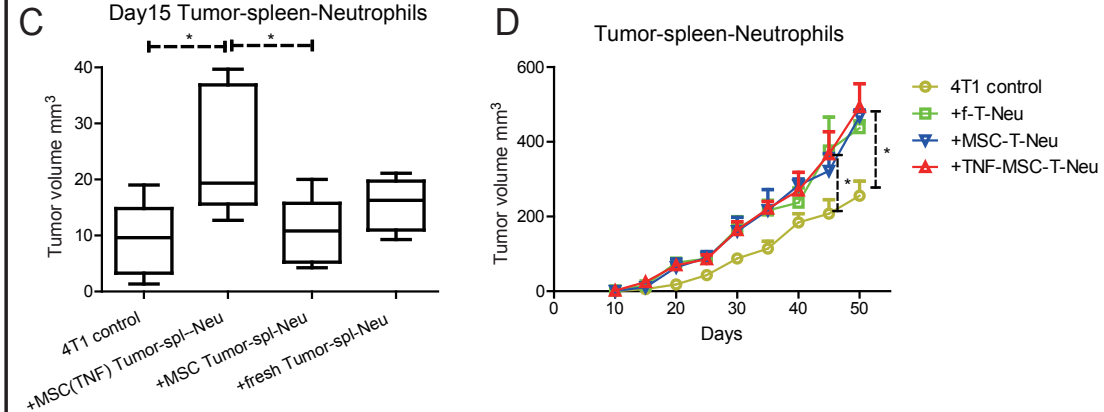
experiments. The tumor volume of tumor-bearing mice treated by f-T-Neu, MSCs-T-Neu, or TNF-MSCs-T-Neu at day $15(\mathrm{C})$, and at the indicated time points (D). Bars represent average \pm SEM. ${ }^{*} p<0.05$ vs $4 \mathrm{~T} 1$ control, ${ }^{*} p<0.05$ vs + MSCs Tumor-spl-Neu and 5 mice per group were used. Data are representative of three independent experiments.

MSCs-Neu. However, compared with MSCs-Neu, TNF-MSC-Neu showed stronger suppressive ability concerning proliferation of $\mathrm{CD}^{+} \mathrm{T}$ cell judged from CFSE dilution and decreased expression of the activation marker $\mathrm{CD} 25$ on both $\mathrm{CD} 4^{+}$and $\mathrm{CD} 8^{+} \mathrm{T}$ cells, all of which indicated the suppression of $\mathrm{T}$ cell activation (Fig. 2A).

We further evaluated the effect of MSCs on the function of neutrophlis from spleens of 4T1 breast tumor-bearing mice. Flow cytometry analysis showed that neutrophils freshly sorted from tumor-bearing mice spleen (f-T-Neu) demonstrated a remarkable suppressive function, reducing FSC and the activation marker CD25 (Fig. 2B). Compared with f-T-Neu, neutrophils coculutred with MSC (MSCs-T-Neu) did not display stronger suppressive effect regarding any markers (Fig. 2B), suggesting that tumor associated neutrophils rather retain than enhance their suppressive function during cocluturing with unprimed MSCs. Strikingly, when neutrophils from spleen of tumor-bearing mice were cocultured with TNF- $\alpha$ primed MSCs (TNF-MSCs-T-Neu), which mimic the modulation of MSCs by tumor microenvironment, these spleen neutrophils displayed much stronger suppressive effect on the activation and proliferation of both $\mathrm{CD}^{+}{ }^{+}$and $\mathrm{CD}^{+}{ }^{+}$cells, with most cell stayed undivided, and obvious down-regulation of CD25 and CD69, and a much lower FSC value (Fig. 2B). Consistently, those cells were very small and few cells clustered together under microscopy, indicating their un-activated status.

These results suggest that there was a significant reduction of anti-CD3 induced T-cell activation and proliferation when adding fresh $\mathrm{CD} 11 \mathrm{~b}^{+} \mathrm{Ly}^{6} \mathrm{G}^{+}$neutrophils sorted from tumor spleen. More importantly, after being cocultured with TNF-MSCs, the suppressive capacity of these $\mathrm{CD} 11 \mathrm{~b}^{+} \mathrm{Ly}_{6} \mathrm{G}^{+}$cells, from BM of normal mice and spleen of tumor bearing mice, was greatly enhanced.

\section{MSC treated neutrophils promote tumor progression in vivo}

4T1 murine breast tumor model was used to explore whether MSC pretreated neutrophils could affect tumor progression in vivo. BM $\mathrm{CD} 11 \mathrm{~b}^{+} \mathrm{Ly}_{6} 6 \mathrm{G}^{+}$neutrophils were 
Fig. 4. Arginase activity was increased in neutrophils after MSC treatment. Neutrophils were cultured alone or cocultured with MSCs, or TNF-MSCs for 72 hours, and measured for arginase activity. Data are representative of two independent experiments.

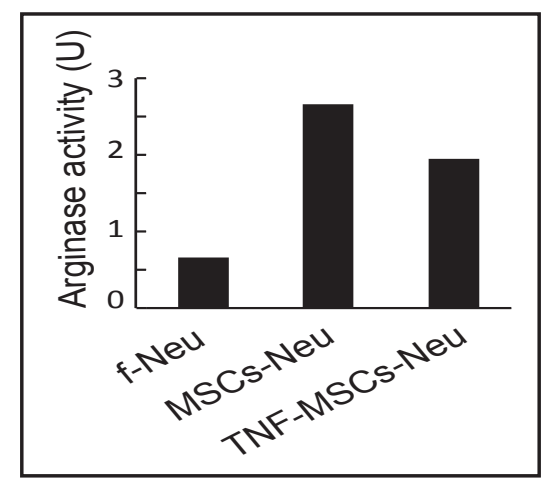

Table 1. Genes with highest fold change in microarray analysis. Genes with highest fold change or related to immune regulation according to microarray analysis between different groups were listed

\begin{tabular}{|c|c|c|c|c|c|c|c|}
\hline \multicolumn{2}{|c|}{ TNF-MSCs-Neu / f-Neu } & \multicolumn{2}{|c|}{ TNF-MSCs-T-Neu / f-T-Neu } & \multicolumn{2}{|c|}{ f-T-Neu / f-Neu } & \multicolumn{2}{|c|}{$\begin{array}{l}\text { TNF-MSCs-T-Neu } \\
\text { /TNF-MSCs-Neu }\end{array}$} \\
\hline $\begin{array}{c}\text { Gene } \\
\text { (mRNA) }\end{array}$ & $\begin{array}{c}\text { Array } \\
\text { (fold change) }\end{array}$ & $\begin{array}{c}\text { Gene } \\
\text { (mRNA) }\end{array}$ & $\begin{array}{c}\text { Array } \\
\text { (fold change) }\end{array}$ & $\begin{array}{c}\text { Gene } \\
\text { (mRNA) }\end{array}$ & $\begin{array}{c}\text { Array } \\
\text { (fold change) }\end{array}$ & $\begin{array}{c}\text { Gene } \\
\text { (mRNA) }\end{array}$ & $\begin{array}{c}\text { Array } \\
\text { (fold change) }\end{array}$ \\
\hline Nos2 & 28 & Fcrls & 112 & Saa3 & 262 & CD209f & 54 \\
\hline Gpnmb & 23 & Dab2 & 72 & Xist & 62 & Fcrls & 42 \\
\hline C3ar1 & 18 & Ms4a7 & 66 & Lipg & 28 & Xist & 31 \\
\hline Saa3 & 14 & $\mathrm{Cd} 209 \mathrm{f}$ & 60 & Dpep2 & 23 & Cxcl13 & 29 \\
\hline Cxcl3 & 14 & Ccl8 & 43 & Nos2 & 17 & Ccl8 & 28 \\
\hline Cxcr1 & 11 & Cxcl13 & 33 & Tlr1 & 8 & Saa3 & 14 \\
\hline Ccl4 & 5 & Ccl12 & 19 & Il13ra1 & 5 & Cd209g & 10 \\
\hline Fas & 5 & Cxcl12 & 9 & Igj & 5 & Fcgrt & 8 \\
\hline Tnf & 4 & Cd209g & 8 & IL-1a & 5 & Ccr3 & 7 \\
\hline Icam1 & 4 & Ccl7 & 8 & Ccr 2 & 5 & $\mathrm{Cd} 4$ & 5 \\
\hline $\mathrm{Ccl} 7$ & 3 & Cxcl16 & 7 & Socs 3 & 4 & Ly86 & 5 \\
\hline Tlr6 & 3 & Fcgr1 & 7 & Il1rap & 4 & Il5ra & 5 \\
\hline Mmp27 & 3 & Il1rl2 & 6 & Ccl2 & 4 & Fcer1a & 5 \\
\hline Sod2 & 3 & Ccr5 & 6 & IL-4ra & 4 & Cxcl16 & 5 \\
\hline Mmp27 & 3 & IL10 & 4 & Cxcr1 & 3 & IL-10 & 4 \\
\hline $\mathrm{Cd} 302$ & 3 & Ccl9 & 4 & IL-10ra & 3 & Ccl12 & 4 \\
\hline IL-1a & 2 & Fas & 4 & IL23a & 3 & Ccl5 & 4 \\
\hline IL-10ra & 2 & IL-6 & 2 & Sod2 & 2 & IL-6 & 4 \\
\hline Il1f8 & 2 & Cxcl5 & 2 & Cxcr5 & 2 & Fn 1 & 3 \\
\hline Arg2 & 2 & Ccl5 & 2 & Ccl9 & 2 & Cxcl5 & 2 \\
\hline
\end{tabular}

cocultured with MSCs for 3 days. Then fresh BM neutrophils (f-Neu), or neutrophils precocultured with MSCs (MSCs-Neu) or TNF- $\alpha$ primed MSCs (TNF-MSCs-Neu) for 3 days, were co-administered with 4T1 cells (4T1:neutrophils $=1: 1$ ) into the mammary gland fat pad of Balb/c mice. And on days 4 and 8, we re-injected neutrophils of different treatments two more times at the same dose in the same position of mice. Tumor size was measured at the indicated time points post inoculation.

Tumor size in MSCs-Neu group and TNF-MSCs-Neu group were larger than f-Neu group and control group. In the early stage (before day 5), the group which was injected with MSCsNeu developed small tumor clumps which could be felt by hand, yet 4T1 control group as well as the group injected f-Neu did not grown detectable tumors at all. During the growth of tumor, the difference between each group became more obvious (Fig. 3A and 3B). However, there was no statistically significant difference between MSCs-Neu group and TNF-MSCsNeu group, or between f-Neu and control group (Fig. 3B). 
Fig. 5. Alteration of critical immune related genes revealed by microarray analysis in neutrophils after MSC treatment. Important genes that were altered in (A) TNF-MSCs-Neu vs f-Neu. (B) TNF-MSCs-T-Neu vs f-TNeu. (C) f-T-Neu vs f-Neu. (D) TNFMSCs-T-Neu vs TNF-MSCs-Neu. Bars represent average \pm SEM. Data are representative of three independent experiments.

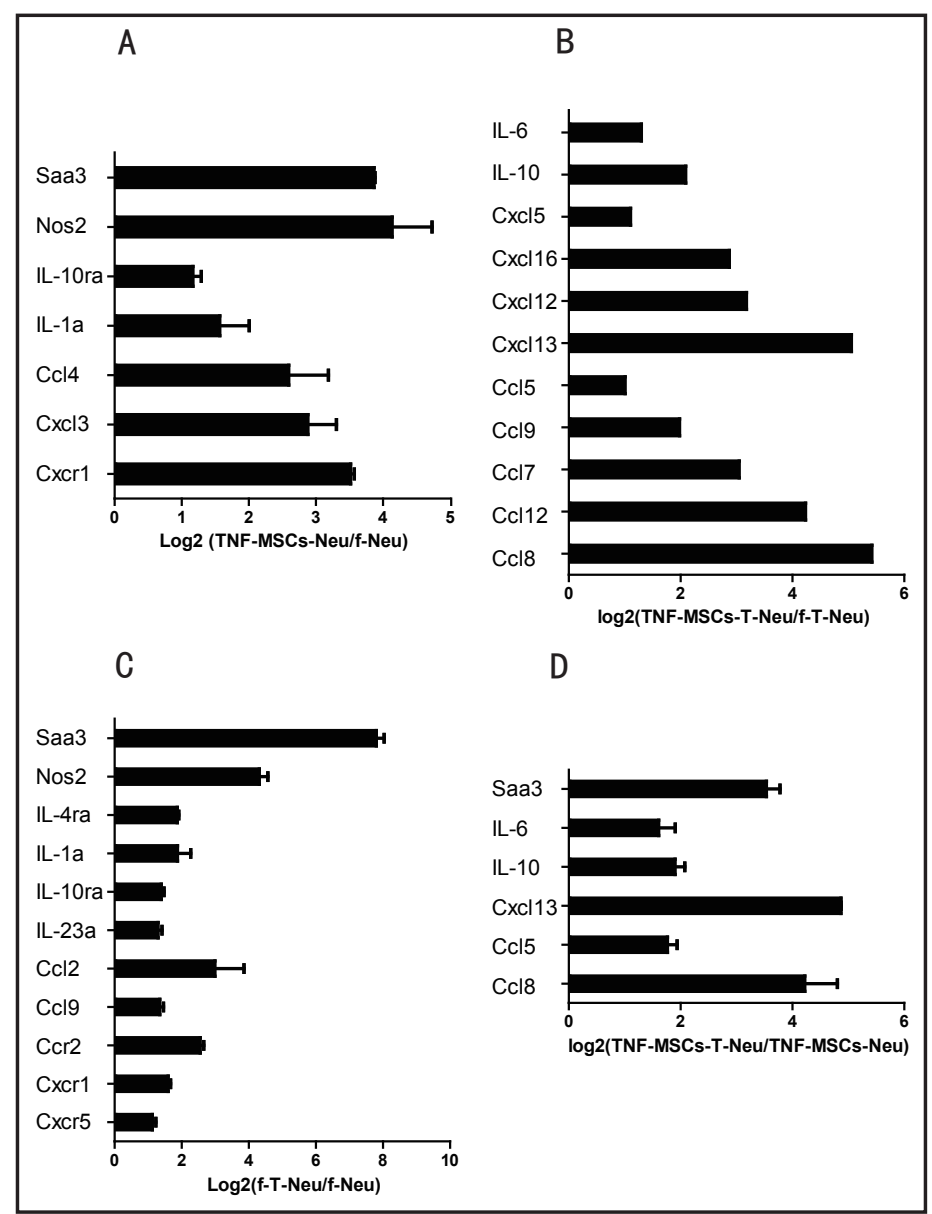

It was then tested whether MSCs could promote the neutrophils from tumor bearing mice spleen to facilitate tumor progression. As described above, 4T1 alone or with f-T-Neu, MSCs-T-Neu, TNF-MSCs-T-Neu were injected into Balb/C mice. TNF-MSCs-T-Neu, MSCs-T-Neu and f-T-Neu groups grew much faster than the control 4T1 group (Fig. 3D). Remarkably, TNFMSCs-Neu exerted stronger effect in facilitating tumor growth in earlier days (before day 15) than later days (Fig. 3C).

\section{MSCs induced arginase activity in neutrophils}

Previous studies show that arginase activity plays an important role in suppressive fuction of neutrophils $[16,21]$. In the present study, arginase activity was also tested in TNFMSCs-Neu, MSCs-Neu and f-Neu. Arginase activity in Neutorphils treated by MSCs was higher than f-Neu. Beyond the expectation is that TNF-MSCs-Neu did not display further increase in arginase activity compared with MSCs-Neu (Fig. 4). These results suggest that not only arginase but also other factors may participate in inducing the suppressive phenotype of neutrophils.

\section{Transcriptional profiling in MSCs-treated neutrophils}

Genome-wide transcriptional profiling analysis was conducted to explore the mechanisms by which MSCs regulate the function of neutrophils. Since TNF- $\alpha$ primed MSCs demonstrated the strongest effect in modulating neutrophil function, BM neutrophils cocultured with TNF- $\alpha$ primed MSCs (TNF-MSCs-Neu) for 40 hours, with fresh BM neutrophils (f-Neu) as control, were subjected to mRNA microarray analysis. Among 2065 genes that expressed differently, 761 were up-regulated and 1304 down-regulated in TNF-MSCs-Neu compared with f-Neu. The top 5 up-regulated genes in TNF-MSCs-Neu were Gpnmb, C3ar1, Nos2, Saa3 and Cxcr1 that were changed by more than 10 -fold (Table 1). It is interesting to 


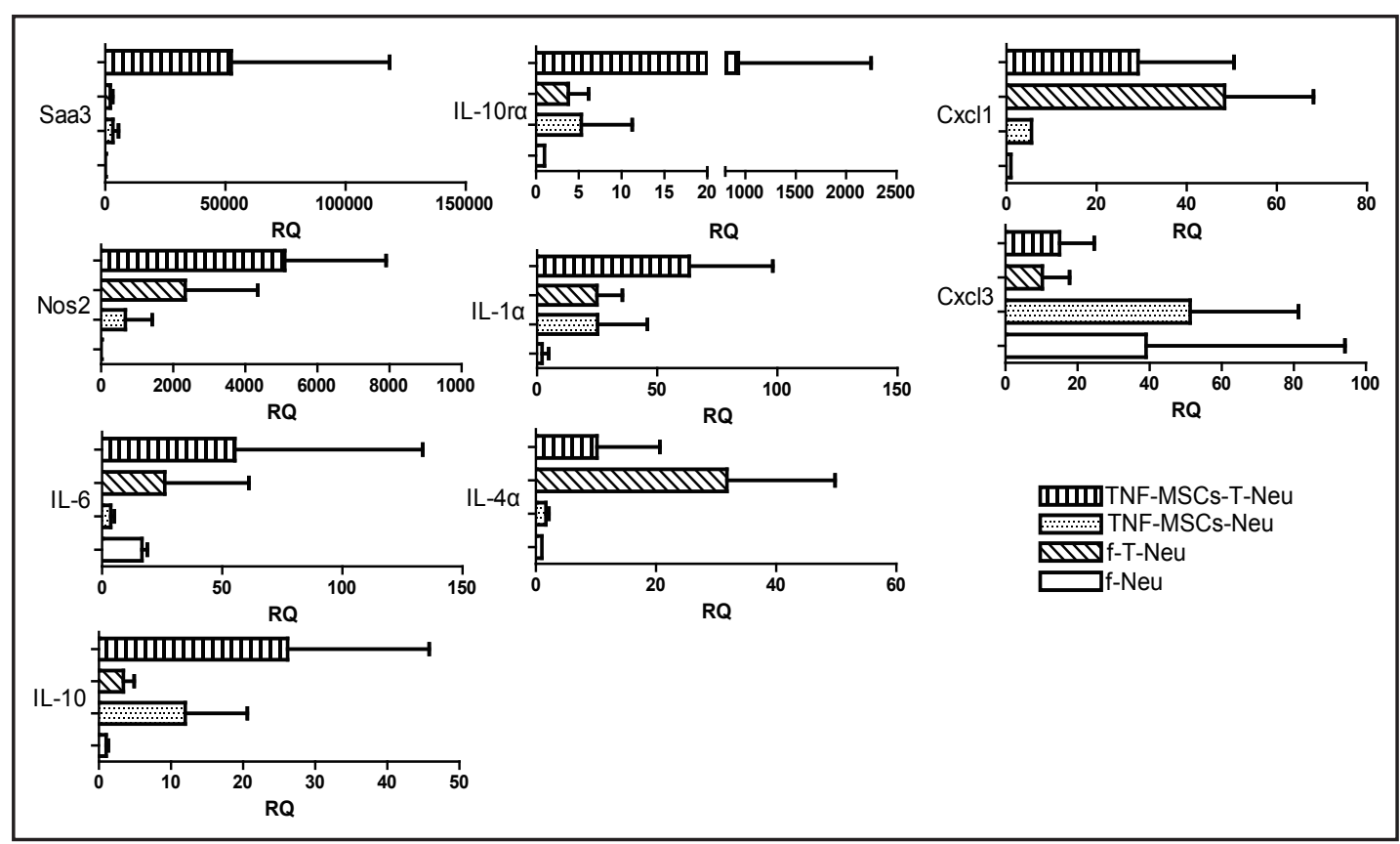

Fig. 6. Confirmation of microarray analysis results by RT-PCR. Real-time RT-PCR was conducted to compare mRNA levels of selected critical genes between f-Neu, f-T-Neu, TNF-MSCs-Neu and TNF-MSCs-T-Neu groups. Bars represent average \pm SEM. Data are representative of two independent experiments.

find a bunch of critical immunosuppression and chemotaxis related genes were increased more than 2 fold, such as Nos2, Saa3, IL-1a, IL-10ra, Cxcr1, Cxcl3, Ccl4 (Fig. 5A).

1011 genes were up-regulated and 483 down-regulated in MSCs-T-Neu compared with f-T-Neu. The top 5 up-regulated genes were Fcrls, Dab2, Ms4a7, Cd209f, Ccl8, all by more than 40-fold (Table 1). Many chemokines and inhibitory cytokines were changed more than 2 fold, such as Ccl8, Cxcl13, Ccl12 Cxcl12, Ccl7, Cxcl16, IL10, Ccl9, Fas, IL-6, Cxcl5, Ccl5 (Fig. 5B).

Fresh BM neutrophils (f-Neu) displayed a very weak immunosuppressive effect, yet the fresh tumor-spleen neutrophils (f-T-Neu) displayed relatively stronger immunosuprressive activity even without pre-coculture with MSCs. 540 genes were up-regulated and 283 downregulated in f-T-Neu compared with $\mathrm{f}$ - Neu. The top 5 up-regulated genes were Saa3, Xist, Lipg, Dpep2, Nos2, all by more than 15 fold (Table 1). Additionally, IL-1a, Ccl2, IL-4ra, Cxcr1, IL-10ra, IL23a, Ccr2, Sod2, Cxcr5, Ccl9 mRNA were up-regulated more than 2 fold. (Fig. 5C).

Finally we found that 906 genes were up-regulated and 167 were down-regulated in TNF-MSCs-T-Neu compared with TNF-MSCs-Neu. The top 5 up-regulated genes were CD209f, Fcrls, Xist, Cxcl13, Ccl8, all by more than 25-fold (Table 1). The most relevant immunosuppressive genes that were changed more than 2 fold were Cxcl13, Ccl8, Saa3, IL10, Ccl5, IL-6 (Fig. 5D).

Validation of gene array results by real-time RT-PCR

The critical genes that were significantly changed in microarray analysis were validated by real-time PCR. The results were highly concordant between the real-time PCR and microarray analysis (Fig. 6), although some small differences in the extent of change were found, possibly due to technical differences between the methods. These results indicate that in various circumstances, MSCs exert complicated impact on neutrophils by altering expression of critical immune related genes including Saa3, Nos2, inhibitory cytokines (IL10), chemokines (Ccl5, Cxcl13) and receptors (IL-10R, IL-4R), which might be closely related to the induction of immunosuppressive and tumor-promoting phenotype. 


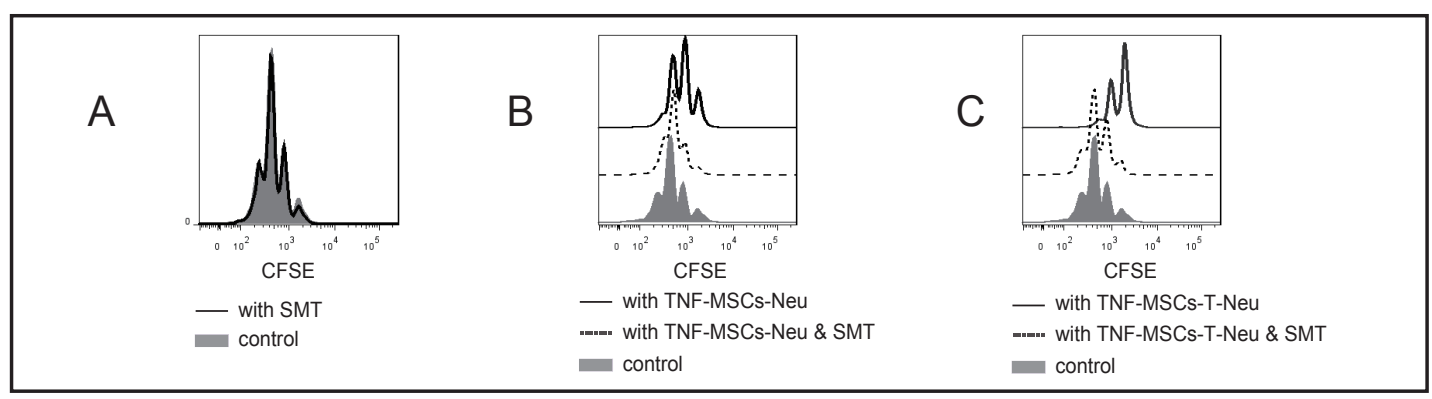

Fig. 7. Roles of increased iNOS activity in suppressive function of TNF-MSC treated neutrophils. (A) Proliferation of anti-CD3 stimulated lymphocytes with or without addition of SMT. (B) Proliferation of anti-CD3 stimulated lymphocytes which were co-cultured with TNF-MSCs-Neu (A) or TNF-MSCs-T-Neu (B) with or without SMT, as assessed by CFSE dilution on day 3. Data are representative of two independent experiments.

\section{Roles of increased iNOS activity in suppressive function of neutrophils}

Since Argniase activity and iNOS are elevated in neutrophils after TNF-MSC treatment, we tested whether inhibition of arginase or iNOS activity could attenuate their suppression function. Lymphocytes stimulated by anti-CD3 antibodies were co-cultured with TNF-MSCsNeu or TNF-MSCs-T-Neu with or without treatment by L-Norvaline (an arginase inhibitor), or SMT (an iNOS inhibitor). Unexpectedly, the Arginase inhibitor L-Norvaline itself could exert strong inhibitive effect on T cell activation and proliferation (data not shown). So it is not feasible to add L-Norvaline to the neutrophil and T cell co-culture system to clarify whether neutrophils exert immunosuppressive function on T cells via arginase activity. Then we tried to pretreat neutrophils with L-Norvaline before co-culturing with T cells to exclude the direct effect of L-Norvaline on T cells. However there were no sufficient neutrophils available for subsequent experiment, since that pretreatment with L-Norvaline led to substantial death of cells. By contrast, SMT alone did not affect the proliferation of $\mathrm{T}$ cell stimulated with anti-CD3 (Fig. 7A). Strikingly, T-cell-suppression function of TNF-MSCs-Neu and TNF-MSCs-T-Neu were greatly impaired by SMT judged by CFSE dilution analysis (Fig. 7B and C). Similar results were obtained regarding $\mathrm{CD}^{+} \mathrm{T}$ cell activation (data not shown). These results indicated although the effect of arginase activity could not be excluded, the increased iNOS activity plays a critical role in the suppressive function of TNF-MSC treated neutrophils.

\section{Discussion}

This study demonstrated that MSCs, especially after TNF- $\alpha$ priming, could program BM neutrphils into an immunosuppressive phenotype which could favor tumor progression. That MSCs induced suppressive neutrophils is similar to the current concept that the granulocytemyeloid derived suppressor cells (G-MDSCs) exist in tumor microenvironment. It has been reported that arginase is critically involved in mechanisms of immune suppression by G-MDSCs [16, 21]. Consistently, our data demonstrated MSCs up-regulated arginase activity in neutrophils. Although any change of arginase-1 expression in microarray analysis was not observed, the mRNA level of arginase-2 changes was higher in TNF-MSCs-Neu than f-Neu, suggesting MSCs may promote arginase activity by up-regulating expression of arginase- 2 .

Transcriptional profiling analysis revealed several critical molecules which might link to the suppressive function of TNF-MSCs-Neu and TNF-MSCs-T-Neu: (i) increased expression of the suppressive factors Nos2 and Saa3, whose activities are always connected with T cell suppression. Saa3 is one of the ligands known to activate TLR-4 signaling, and elevation of TLR-4 is involved in promoting tumor progression [22]. Recent studies show that G-MDSCs impair T cell responses through nitric oxide (NO) dependent pathways [23]. (ii) increased levels of anti-inflammatory cytokines and their receptors IL-10, IL-6, IL-4R, IL-10R that are 
Fig. 8. Proposed model depicting the interaction between MSCs and neutrophils during tumor development. TNF- $\alpha$, and possibly other mediators, educated MSCs, directly program neutrophils into a suppressive phenotype and promote tumor development. MSCs may participate in the in vivo generation of G-MDSCs in the spleen and their further functional polarization when migrated into the tumor.

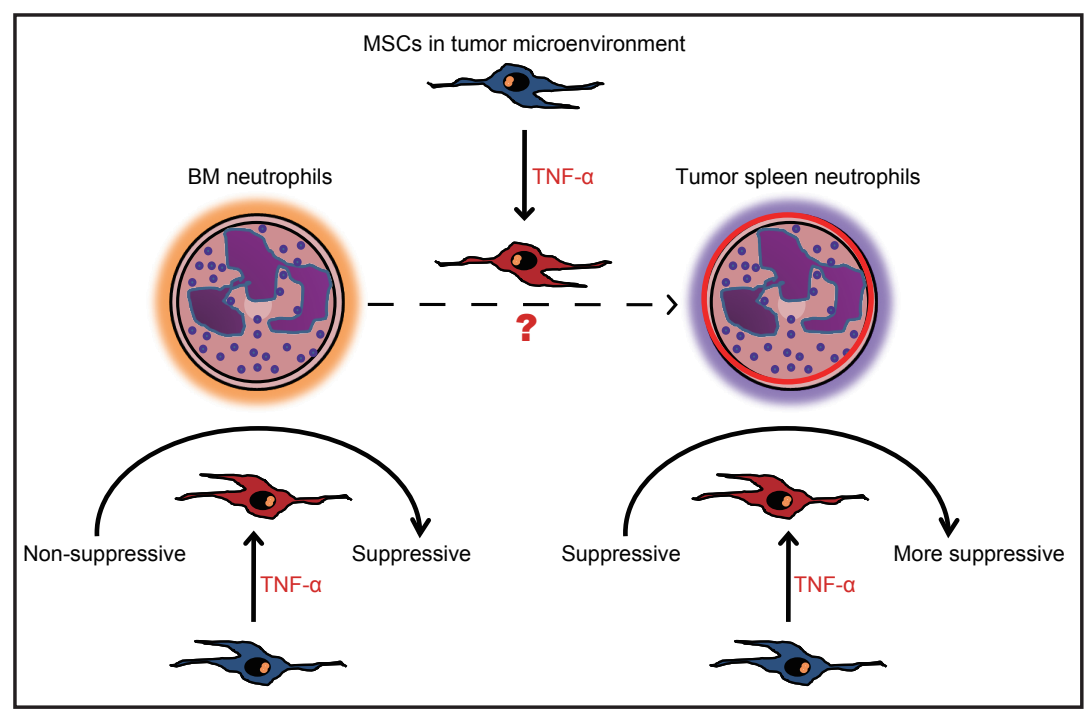

known to promote tumor progression. Previous studies showed that increased IL-6 levels have been associated with elevated cancer risk, and also been found to be a prognostic factor for several cancer types [24]. IL-10 is emerging as a key cytokine mediating the suppressive function of neutrophils, and dampening tumor-specific immune surveillance [25-27]. IL4R $\alpha$-STAT6 signaling could induce the production of ARG1 by MDSCs [28]. In addition, the IL-4R $\alpha$-STAT6 pathway was also found to be involved in IL-13-induced TGF- $\beta$ production by MDSCs in mice with sarcoma, which resulted in decreased tumor immunosurveillance [29]. (iii) increased production of the chemokines Cxcl13, Ccl8 etc., which might result in further recruitment of suppressive immune cells to the tumor microenvironment to favor tumor growth.

Actually out data showed that the immunosuppressive function of neutrophils was markedly impaired by a selective iNOS inhibitor SMT, supporting that the suppressive function of neutrophils treated by TNF- $\alpha$ primed MSCs, is mediated at least in part, via increased iNOS activity and NO production.

Previous studies showed that TNF- $\alpha$ plays a major role in the enhancement of tumor progression by modulating multiple tumor-related cell types, and TNF- $\alpha$ treated BM MSCs displayed a function and profile of cytokine/chemokine production resembling that of tumor-resident MSCs [9]. TNF- $\alpha$ educated MSCs could enhance tumor growth via monocyte/ macrophage recruitment [9]. In our study, neutrophils cocultured with TNF- $\alpha$ primed MSCs showed a stronger suppressive function than that cocultured with unprimed MSCs. Furthermore, TNF-MSCs-Neu seems to exert stronger effect on promoting early tumor growth. Thus our data support that TNF- $\alpha$ educated MSCs may favor tumor progression by directly promoting the suppressive function of neutrophils.

We also found that neutrophils freshly isolated from spleen of tumor bearing mice, which agree with the established concept of G-MDSCs, displayed substantial capacity to suppress T cell proliferation and favor tumor progression, contrasted by the marginal effect of normal BM neutrophils. Actually, IL-10, Nos2, and arginase are reported to be directly involved in mechanisms of immune suppression by G-MDSCs. Strikingly, our data demonstrated all the three molecules were dramatically higher in either TNF-MSC-neutrophils or tumor-spleen neutrophils, than nomal BM neutrophils, This results implicated that tumor-spleen-derived neutrophils might have already been educated to transform into immunosuppressive phenotype by mesenchymal stromal cells in the tumor bearing host. Nevertheless in vitro coculture with TNF- $\alpha$ primed MSC further potentiate the T-cell-suppressive and tumorpromoting effect of tumor-spleen neutrophils, suggesting that in vivo, tumor associated MSCs may play a crucial role in promoting not only the development of G-MDSCs in the 
spleen, but also their further functional polarization inside the tumor mass during tumor development.

Taken together our study revealed a novel mechanism of promotion of tumor progression by linking the interaction between MSC and neutrophils. Based on our findings, we propose the following model (Fig. 8). During tumor development, BM mobilized or tissue resident MSCs are polarized to an activation status by tumor-elicited factors including TNF- $\alpha$. These activated MSCs promoted survival of neutrophils and program them into an immunosuppressive phenotype to favor tumor development. Our study suggested that strategies that target MSC-neutrophil crosstalk might provide a novel avenue of cancer therapy, and furthermore, MSC transplantation therapy should be carried out with special caution in cancer patients.

\section{Acknowledgments}

This work was supported by National Basic Research Program of China (2013CB966904), National Natural Science Foundation of China (81273217, 81170007, 81370104), Tianjin Research Program of Application Foundation and Advanced Technology (12JCYBJC32800), Specialized Research Fund for the Doctoral Program of Higher Education of China (20121106120037), and The Recruitment Program of Global Youth Experts.

\section{Disclosure Statement}

The authors declare that they have no competing financial interests.

\section{References}

1 English K, French A, Wood KJ: Mesenchymal stromal cells: Facilitators of successful transplantation? Cell Stem Cell 2010;7:431-442.

- Mendez-Ferrer S, Michurina TV, Ferraro F, Mazloom AR, Macarthur BD, Lira SA, Scadden DT, Ma'Ayan A, Enikolopov GN, Frenette PS: Mesenchymal and haematopoietic stem cells form a unique bone marrow niche. Nature 2010;466:829-834.

- 3 Pittenger MF, Mackay AM, Beck SC, Jaiswal RK, Douglas R, Mosca JD, Moorman MA, Simonetti DW, Craig S, Marshak DR: Multilineage potential of adult human mesenchymal stem cells. Science 1999;284:143-147.

4 Di Nicola M, Carlo-Stella C, Magni M, Milanesi M, Longoni PD, Matteucci P, Grisanti S, Gianni AM: Human bone marrow stromal cells suppress T-lymphocyte proliferation induced by cellular or nonspecific mitogenic stimuli. Blood 2002;99:3838-3843.

-5 Mishra PJ, Mishra PJ, Humeniuk R, Medina DJ, Alexe G, Mesirov JP, Ganesan S, Glod JW, Banerjee D: Carcinoma-associated fibroblast-like differentiation of human mesenchymal stem cells. Cancer Res 2008;68:4331-4339.

-6 Quante M, Tu SP, Tomita H, Gonda T, Wang SS, Takashi S, Baik GH, Shibata W, Diprete B, Betz KS, Friedman R, Varro A, Tycko B, Wang TC: Bone marrow-derived myofibroblasts contribute to the mesenchymal stem cell niche and promote tumor growth. Cancer Cell 2011;19:257-272.

-7 Spaeth EL, Dembinski JL, Sasser AK, Watson K, Klopp A, Hall B, Andreeff M, Marini F: Mesenchymal stem cell transition to tumor-associated fibroblasts contributes to fibrovascular network expansion and tumor progression. PLoS One 2009;4:e4992.

8 Studeny M, Marini FC, Dembinski JL, Zompetta C, Cabreira-Hansen M, Bekele BN, Champlin RE, Andreeff M: Mesenchymal stem cells: Potential precursors for tumor stroma and targeted-delivery vehicles for anticancer agents. J Natl Cancer Inst 2004;96:1593-1603.

-9 Ren G, Zhao X, Wang Y, Zhang X, Chen X, Xu C, Yuan ZR, Roberts AI, Zhang L, Zheng B, Wen T, Han Y, Rabson AB, Tischfield JA, Shao C, Shi Y: CCR2-dependent recruitment of macrophages by tumor-educated mesenchymal stromal cells promotes tumor development and is mimicked by TNFalpha. Cell Stem Cell 2012;11:812-824. 


\section{Cellular Physiology Cell Physiol Biochem 2014;33:1802-1814

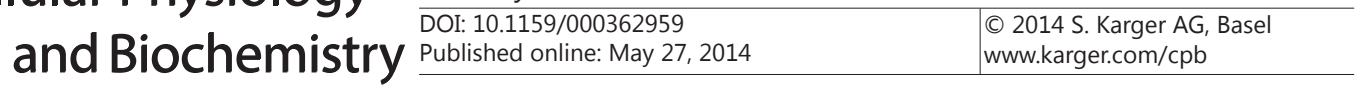

Hu et al.: MSCs Induces Tumor-Promoting Neutrophils

10 Eggenhofer E, Hoogduijn MJ: Mesenchymal stem cell-educated macrophages. Transplant Res 2012;1:12.

11 Nathan C: Neutrophils and immunity: Challenges and opportunities. Nat Rev Immunol 2006;6:173-182.

12 Koenderman L, Yazdanbakhsh M, Roos D, Verhoeven AJ: Dual mechanisms in priming of the chemoattractant-induced respiratory burst in human granulocytes. A Ca2+-dependent and a Ca2+independent route. J Immunol 1989;142:623-628.

13 Hakansson L, Venge P: Priming of eosinophil and neutrophil migratory responses by interleukin 3 and interleukin 5. Apmis 1994;102:308-316.

14 Matsushima H, Geng S, Lu R, Okamoto T, Yao Y, Mayuzumi N, Kotol PF, Chojnacki BJ, Miyazaki T, Gallo RL, Takashima A: Neutrophil differentiation into a unique hybrid population exhibiting dual phenotype and functionality of neutrophils and dendritic cells. Blood 2013;121:1677-1689.

15 Fridlender ZG, Sun J, Kim S, Kapoor V, Cheng G, Ling L, Worthen GS, Albelda SM: Polarization of tumorassociated neutrophil phenotype by TGF-beta: "N1" versus "N2" TAN. Cancer Cell 2009;16:183-194.

-16 Pillay J, Tak T, Kamp VM, Koenderman L: Immune suppression by neutrophils and granulocytic myeloidderived suppressor cells: Similarities and differences. Cell Mol Life Sci 2013;70:3813-3827.

-17 Cassatella MA, Mosna F, Micheletti A, Lisi V, Tamassia N, Cont C, Calzetti F, Pelletier M, Pizzolo G, Krampera M: Toll-like receptor-3-activated human mesenchymal stromal cells significantly prolong the survival and function of neutrophils. Stem Cells 2011;29:1001-1011.

18 Peister A, Mellad JA, Larson BL, Hall BM, Gibson LF, Prockop DJ: Adult stem cells from bone marrow (MSCs) isolated from different strains of inbred mice vary in surface epitopes, rates of proliferation, and differentiation potential. Blood 2004;103:1662-1668.

19 Urban VS, Kiss J, Kovacs J, Gocza E, Vas V, Monostori E, Uher F: Mesenchymal stem cells cooperate with bone marrow cells in therapy of diabetes. Stem Cells 2008;26:244-253.

20 Feng X, Ippolito GC, Tian L, Wiehagen K, Oh S, Sambandam A: L Foxp1 is an essential transcriptional regulator for the generation of quiescent naive T cells during thymocyte development. Blood 2010;115:510-518

21 Rodriguez PC, Quiceno DG, Ochoa AC: L-arginine availability regulates T-lymphocyte cell-cycle progression. Blood 2007;109:1568-1573.

22 Hiratsuka S, Watanabe A, Sakurai Y, Akashi-Takamura S, Ishibashi S, Miyake K, Shibuya M, Akira S, Aburatani H, Maru Y: The S100A8-serum amyloid A3-TLR4 paracrine cascade establishes a pre-metastatic phase. Nat Cell Biol 2008;10:1349-1355.

23 Raber PL, Thevenot P, Sierra R, Wyczechowska D, Halle D, Ramirez ME, Ochoa A, Fletcher M, Velasco C, Wilk A, Reiss K, Rodriguez PC: Subpopulations of Myeloid-Derived Suppressor Cells (MDSC) impair T cell responses through independent nitric oxide-related pathways. Int J Cancer 2014;134:2853-2864.

-24 Zarogoulidis P, Yarmus L, Darwiche K, Walter R, Huang H, Li Z, Zaric B, Tsakiridis K, Zarogoulidis K: Interleukin-6 cytokine: A multifunctional glycoprotein for cancer. Immunome Res 2013;9:16535.

25 Noel G, Wang Q, Schwemberger S, Hanson C, Giacalone N, Haar L, Ogle CK: Neutrophils, not monocyte/ macrophages, are the major splenic source of postburn IL-10. Shock 2011;36:149-155.

-26 De Santo C, Arscott R, Booth S, Karydis I, Jones M, Asher R, Salio M, Middleton M, Cerundolo V: Invariant NKT cells modulate the suppressive activity of IL-10-secreting neutrophils differentiated with serum amyloid a. Nat Immunol 2010;11:1039-1046.

27 Davey MS, Tamassia N, Rossato M, Bazzoni F, Calzetti F, Bruderek K, Sironi M, Zimmer L, Bottazzi B, Mantovani A, Brandau S, Moser B, Eberl M, Cassatella MA: Failure to detect production of IL-10 by activated human neutrophils. Nat Immunol 2011;12:1017-1018, 1018-1020.

28 Sinha P, Clements VK, Ostrand-Rosenberg S: Interleukin-13-regulated M2 macrophages in combination with myeloid suppressor cells block immune surveillance against metastasis. Cancer Res 2005;65:1174311751.

29 Terabe M, Matsui S, Park JM, Mamura M, Noben-Trauth N, Donaldson DD, Chen W, Wahl SM, Ledbetter S, Pratt B, Letterio JJ, Paul WE, Berzofsky JA: Transforming growth factor-beta production and myeloid cells are an effector mechanism through which CD1d-restricted T cells block cytotoxic T lymphocyte-mediated tumor immunosurveillance: Abrogation prevents tumor recurrence. J Exp Med 2003;198:1741-1752. 